\title{
PROCESSING PIPELINE FOR DIGITALIZING THE LINEAGE TREE OF EARLY ZEBRAFISH EMBRYOGENESIS FROM MULTIHARMONIC IMAGING
}

\author{
Miguel A Luengo-Oroz ${ }^{\star}$, Thierry Savy ${ }^{\dagger}$, Jose L Rubio ${ }^{\star}$, Louise Duloquin ${ }^{\dagger}$, \\ Emmanuel Faure ${ }^{b}$, Nicolas Olivier $\sharp$, Maria Ledesma-Carbayo ${ }^{\star}$, Delphine Debarre ${ }^{\sharp}$, \\ Paul Bourgine ${ }^{\downarrow}$ Emmanuel Beaurepaire ${ }^{\sharp}$, Nadine Peyrieras ${ }^{\dagger}$, Andres Santos ${ }^{\star}$ \\ * Biomedical Image Technologies, ETSI Telecomunicacion, Universidad Politecnica de Madrid, Spain \\ ${ }^{b}$ Centre de Recherche en Epistemologie Appliquee, Ecole Polytechnique, CNRS, Paris, France \\ ${ }^{\dagger}$ Neurobiologie et Developpement, Institut de Neurobiologie Alfred Fessard, CNRS, Gif/Yvette, France \\ \# Laboratory for Optics and Biosciences, Ecole Polytechnique, CNRS, INSERM, Palaiseau, France
}

\begin{abstract}
The reconstruction of the cell lineage tree of early zebrafish embryogenesis requires the use of in-vivo microscopy imaging and image processing strategies. Second (SHG) and third harmonic generation (THG) microscopy observations in unstained zebrafish embryos allows to detect cell divisions and cell membranes from 1-cell to $1 \mathrm{~K}$-cell stage. In this article, we present an ad-hoc image processing pipeline for cell tracking and cell membranes segmentation enabling the reconstruction of the early zebrafish cell lineage tree until the $1 \mathrm{~K}$-cell stage. This methodology has been used to obtain digital zebrafish embryos allowing to generate a quantitative description of early zebrafish embryogenesis with minute temporal accuracy and $\mu m$ spatial resolution [1].
\end{abstract}

Index Terms - in-vivo imaging, lineage tree, multiphoton microscopy, viscous watershed, zebrafish development

\section{INTRODUCTION}

Image processing methods are a critical step for the analysis of embryogenesis processes based on in-vivo microscopy imaging observations [2]. Achieving the digital reconstruction of the cell lineage tree of model organisms such as zebrafish is an open challenge in systems biology. The zebrafish is an organism model used for the study of vertebrates in developmental biology with special interest in cancer and stem cells [3]. Zebrafish embryos are transparent, allowing to acquire high resolution in-vivo images of cell nuclei, cell membranes or reporters of gene expression activity during their development. In [4], the first $24 h$ of zebrafish development were reconstructed using light-sheet based fluorescence microscopy. However, detailed quantitative analysis

This work was supported by FPI and ARTEMIS, Comunidad de Madrid (Spain), AMIT (CDTI - Ministry of Science, Spain), Agence Nationale de la Recherche (France), the European Regional Development Funds and the FP6 New Emerging Science and Technology program. of embryos at early stages is difficult because the fluorescent protein expression is either weak or poorly localized in subcellular structures during the first hours post fertilization (hpf). Multiharmonic microscopy is an alternative modality that does not need fluorescence markers because images are produced by the intrinsic properties of the biological matter. Therefore, the acquisition of combined SHG and THG imaging of unstained zebrafish embryos produces $3 D+T$ image sequences allowing to see new biological phenomena during initial divisions cycles, particularly before $3 \mathrm{hpf}$ when zebrafish embryos enter a period which is characterized by cell cycle lengthening, loss of cell division synchrony, activation of transcription and appearance of cell motility. THG images reveal cell membrane contours. Since no THG can be obtained from a homogeneous medium, this signal reflects the presence of a sizeable intercellular space between cells. SHG images reveal the formation of mitotic spindles (see Fig.1) showing a spot during cell division whose intensity exhibits a Gaussian-like temporal behavior, permitting to measure division timings and cell cycle duration. This paper details an $a d-h o c$ image processing pipeline for the reconstruction of the digital zebrafish embryo during the first ten cell cycles using SHG and THG microscopy data.

\section{METHODS OVERVIEW}

The goal of the proposed methodology is to write the individual history of each cell in terms of lineage and shape from the first to the tenth cell cycle, going from to 1 to $1 K$ cells. Input data are SHG and THG time lapses. Intracellular SHG signal exhibits a peak during mitosis that will be used to extract the lineage tree. THG signal highlights membrane geometry and is used to segment the cell shape. A typical acquisition is in the order of 100 volumes of size $(512,512,120)$ voxels with resolution of $(2,2,4) \mu \mathrm{m}^{3}$ and $\Delta T=90 \mathrm{~s}$. Context-specific features have been taken into account for designing the processing pipeline: 1) Visual observation shows that all mitosis 


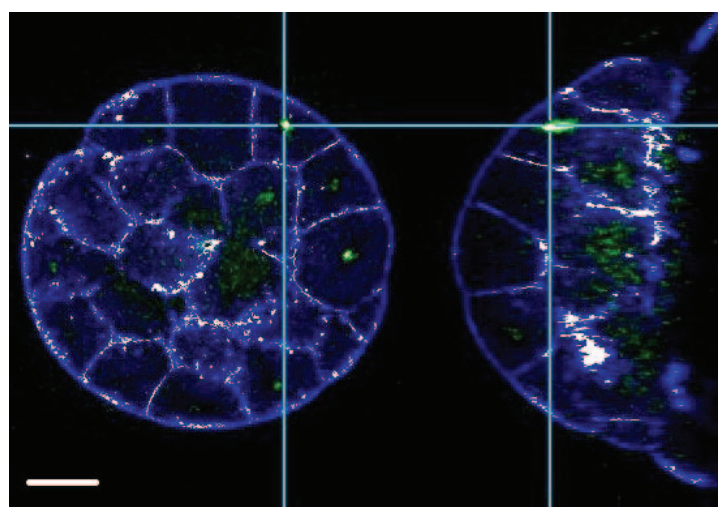

Fig. 1. SHG (green) and THG (blue) imaging of unstained zebrafish embryo at 64-cell stage (scale bar is $100 \mu \mathrm{m}$ ).

during a cell cycle occur almost at the same time (i.e. all the cells from 4 to 8 cells divide almost synchronously and then proceed as a wave through the embryo). 2) During this developmental period, we may assume that cells do not move except for mitosis. 3) During mitosis, daughter cells move symmetrically with respect to their mother cell.

Because errors in mitosis detection propagate along the lineage, the processing pipeline has been coupled with the MovIT validation interface (Savy et al., unpublished). In the proposed procedure, divisions in each cell cycle are automatically detected and manually checked and corrected before analyzing the next cell cycle. This strategy allows minimizing the human intervention to extract correct data. In Fig.2, the processing pipeline is presented. First, the SHG image sequence is divided into subsequences corresponding to each cell cycle and then each subsequence is collapsed into one frame, so time lapses that typically have one-hundred time points are reduced to ten. Next, the lineage tree is extracted from collapsed SHG data (identifying the cell daughters of each cell in the previous cycle). Then SHG spot detection is manually corrected with the Mov-IT interface and the procedure of automatic detection and validation/correction is iterated for all the cycles. Next, the timing of mitosis events is refined using the non-collapsed original complete SHG sequence. Finally, the nuclei position of each cell is used as seed for a viscous watershed segmentation of THG membrane images. In the following sections, all the steps are presented in detail.

\section{PROCESSING PIPELINE}

\subsection{SHG cycle fusion}

First, SHG data is filtered with a 3D median operator and convolved with a gaussian kernel for regularization purposes. Visual inspection of SHG data shows that the mitotic spots of each division cycle are organized in temporal waves across

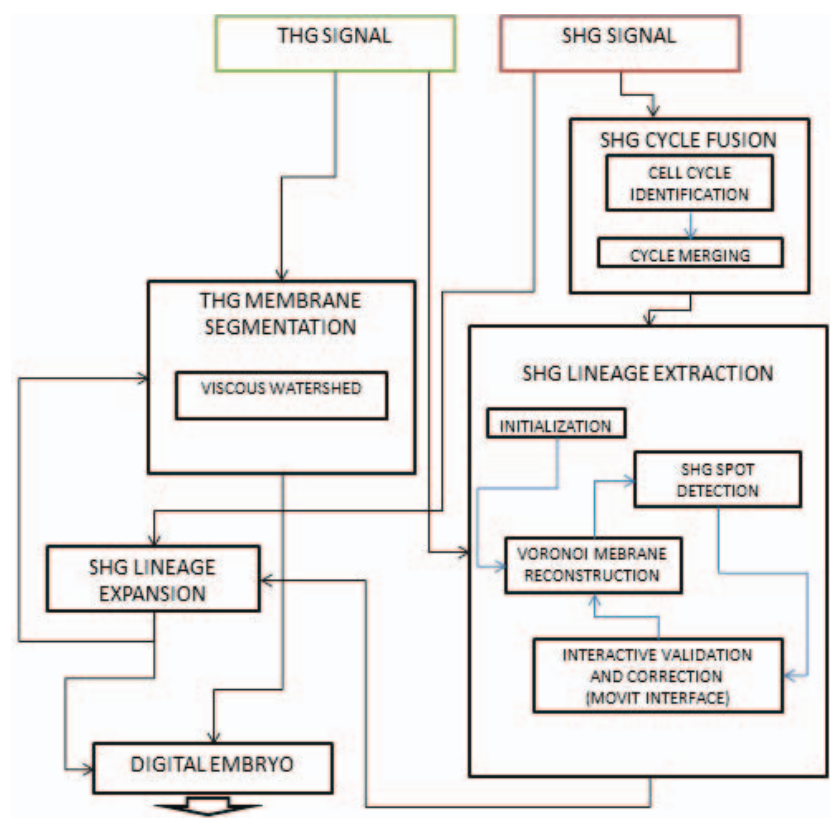

Fig. 2. Schema of the processing pipeline for generating the digital zebrafish embryo from SHG and THG data.

the embryo. The timing of this global mitotic clock can be extracted from the integration of the total SHG signal along time. The measurement total-SHG-signal presents an oscillatory behavior where maxima correspond to the time points where most of the mitosis are occurring inside a division cycle and minima correspond to moments between two division waves. The identification of the function minima allows to find the time intervals corresponding to each cell cycle (see Fig.3). In order to produce a single 3D volume per division

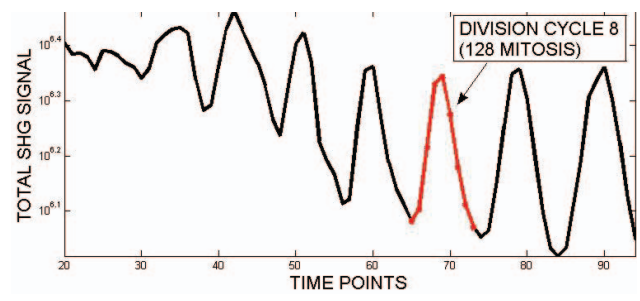

Fig. 3. Total SHG signal intensity along time. Signal peaks correspond to division waves.

cycle, all the time points belonging to a division wave are added into a single 3D image volume (see Fig.4).

\subsection{SHG lineage extraction}

The goal of this module is to identify all the SHG spots in each division cycle and link each cell with its two daughter cells in the next cycle. The inputs of this module are the ten 

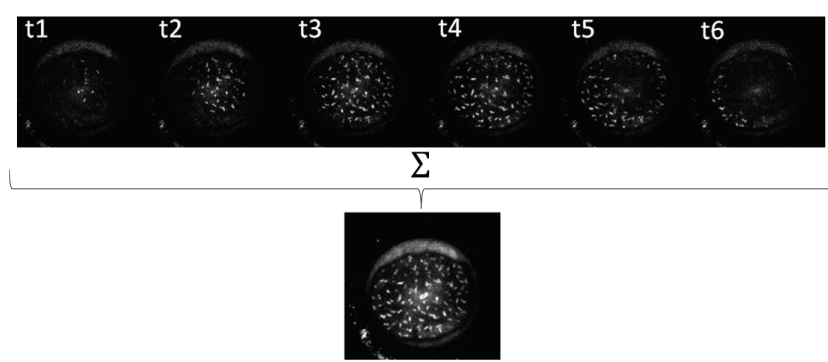

Fig. 4. Projection of consecutive SHG volumes and its resulting collapsed single volume (128-cell stage).

SHG fused images corresponding to the first ten cycles and the ten embryo binary masks (extracted from THG data using mathematical morphology operators). The Voronoi regions inside the embryo mask generated from cell centers are a reasonable approximation to membrane boundaries [5]. Hence, we can assume that when a mother cell divides, the nuclei of the two daughter cells are going to divide inside the Voronoi region associated with their mother cell in the previous cycle. The iterative procedure comprises the following steps: 1) Find the SHG spots corresponding to the first division cycle, typically two if starting with two cells. They are the two highest intensity regional maxima inside the embryo mask. Afterwards the Voronoi partition from the detected spots are created inside the embryo mask of the next division cycle. 2) Find the SHG spots corresponding to the next division cycle (twice the number of cells than in the previous cycle). For each Voronoi region extracted from the previous cycle analysis, the maximum of intensity is found and corresponds to the first daughter cell. During mitosis both daughter cells have symmetric displacements relative to the mother cell, therefore the search for the second daughter is restricted to the symmetric hemisphere inside the Voronoi region with respect to the first daughter and the mother cell. The local intensity maximum in this restricted region is selected as the second daughter cell (see Fig.5). 3) The detected spots are visually validated and corrected using the Mov-IT interface. Error rates in detection before validation are in the order of $10 \pm 5 \%$ (note that the number of false positives equals the false negatives, meaning that correcting a spot simply consist in moving the position - which is easier than creating or deleting spots). 4) The Voronoi partition of the embryo mask in the next division cycle is created from detected spots. The procedure goes back to step 2 until all the cycles are analyzed.

\subsection{SHG lineage temporal expansion}

The previous module allowed the extraction of the cell lineage tree from the collapsed SHG images providing the parental relationships and the spatial position. Nevertheless, it does not show the exact time of mitosis inside the division cycle.
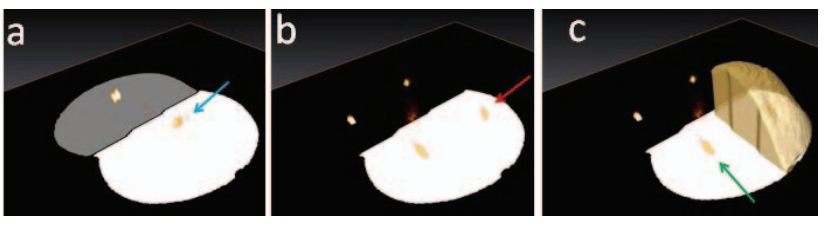

Fig. 5. Illustrative example of mitosis tracking. (a) 2-cell stage spots detection and associated Voronoi regions (in grey and white). Arrow points to the cell that is going to be tracked. (b) Red arrow points to the SHG spot detected in the 4-cell stage inside the Voronoi region of the previously pointed cell (blue arrow). (c) Green arrow points to the second daughter outside the isosurface region showing the forbidden region associated to the other daughter (red arrow).

In order to find the precise timing of mitosis, SHG intensity values at the detected spatial position of each mitosis along consecutive time points, are fitted to a Gaussian function that models SHG signal during mitosis. Thus, the maximum of the fitted function refines the timing of mitosis (see Fig.6 and Fig.7). Moreover, to be coherent with the global timing, the acquisition delay corresponding to each $x y$ slice in the same $3 D$ volume has been also compensated.
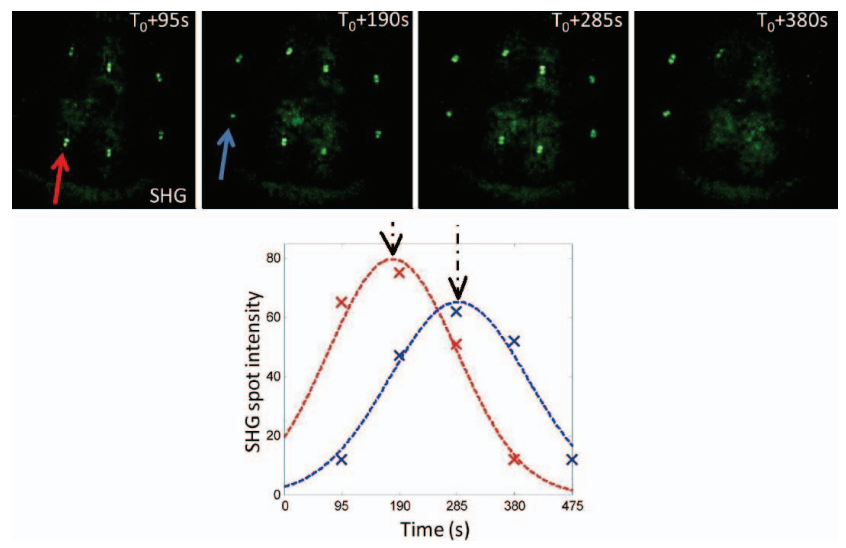

Fig. 6. (top) Four consecutive frames of SHG signal. (bottom) Intensity measurement at the different time steps on 2 cells and the gaussian fitting of SHG signal along time.

\subsection{THG membrane segmentation}

The THG signal serves as a membrane marker that allows extracting cell shape. Cell segmentation is approached by a watershed-based methodology where the seeds are the cell centers extracted from the SHG spots. However, the THG signal delineating cell boundaries is in some places poorly defined, leading to leakage problems with the standard watershed. To address this issue, we have implemented a vis- 

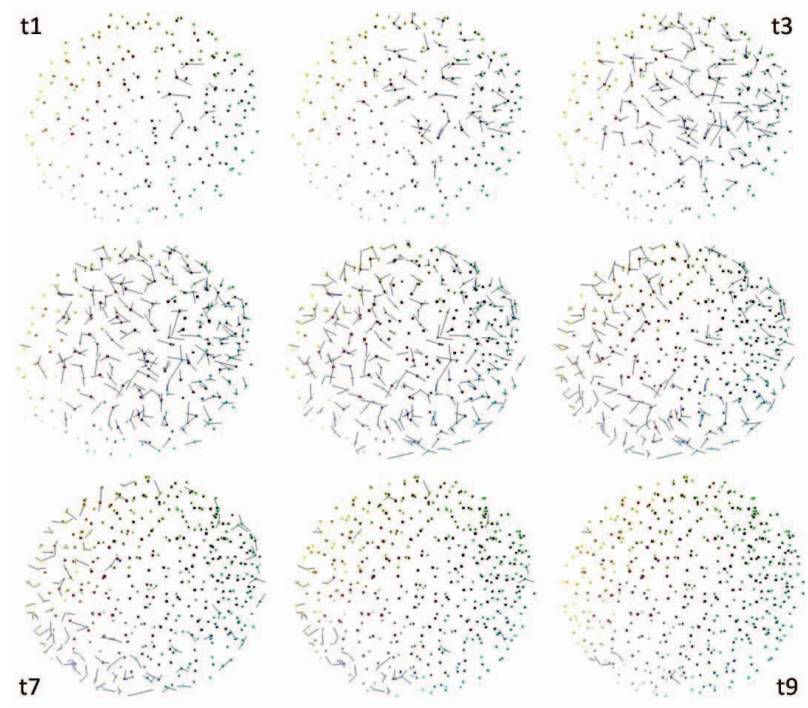

Fig. 7. Nine consecutive frames (view from above) of the cell lineage expanded during the passage from cycle 9 to 10 . The timing of each mitosis is represented by a dipole oriented with the position of the two cell daughters. Mitosis spatiotemporal arrangement shows a wave pattern crossing the embryo lasting these 9 frames.

cous watershed methodology [6] that consists in modifying the topology of the image by means of a viscous closing and then performing the standard watershed. The oil-model fluid has been chosen for the viscous closing transformation and parameters have been chosen empirically. Segmentation accuracy has been validated against a manually segmented gold standard obtained using ITK-Snap showing that the viscous watershed is more accurate and produces less leakage than the standard watershed.

\section{RESULTS AND DISCUSSION}

The presented processing pipeline provides the cell lineage tree annotated with cells position in each time step (corresponding to the nuclei position where the SHG spot signals the mitotic state), the parental relation between cells and the cell shape either calculated with the Voronoi approximation and the viscous watershed segmentation of THG signal. Reconstructed data can be interactively visualized with the MovIT interface. Methods have been written in MATLAB and the whole process for analyzing a complete dataset takes about $72 \mathrm{~h}$ of computation (Core2Duo, 8Gb RAM) and $4 \mathrm{~h}$ of user interaction for manual validation and script launching. Six zebrafish embryos have been completely reconstructed from 2 cells to the $1 K$-cell stage. The previous methodology is limited to the analysis until $1 \mathrm{~K}$-cell stage because later on cells enter a developmental period characterized by mitosis desynchronization and cells intrinsic motility. In summary, a dedicated image processing pipeline for tracking and segmentation of SHG/THG image sequences of early zebrafish development has been designed and implemented. Data analysis of the reconstructed embryos (see Fig. 8) provide a new exhaustive and comprehensive quantitative description of early zebrafish development [1].

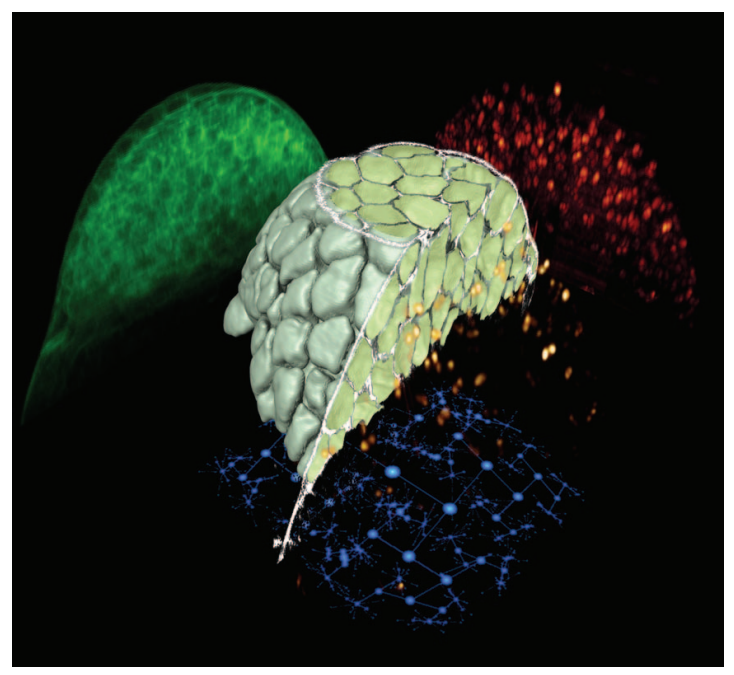

Fig. 8. Zebrafish embryo reconstructed at the 128-cell stage. Volume rendering shows the raw THG signal in white, raw SHG in orange and membrane segmentation in light green. The figure also includes three projections showing the THG signal (green), the SHG signal (red) and a flat projective representation of the cell lineage tree (blue).

\section{REFERENCES}

[1] Olivier et al., "Cell Lineage Reconstruction of Early Zebrafish Embryos Using Label-Free Nonlinear Microscopy,” Science, vol. 329, no. 5994, pp. 967, 2010.

[2] Oates et al., "Quantitative approaches in developmental biology," Nature Rev Gene, vol. 10, pp. 517-530, 2009.

[3] Amatruda et al., "Zebrafish as a cancer model system," Cancer Cell, vol. 1, no. 3, pp. 229-231, 2002.

[4] Keller et al., "Reconstruction of Zebrafish Early Embryonic Development by Scanned Light Sheet Microscopy," Science, vol. 322, no. 5904, pp. 1065, 2008.

[5] Luengo-Oroz et al., "Can voronoi diagram model cell geometries in early sea-urchin embryogenesis?," IEEE ISBI, pp. 504-507, 2008.

[6] C. Vachier and F. Meyer, "The Viscous Watershed Transform," Mathematical Imaging and Vision, vol. 22, no. 2-3, pp. 251-267, 2005. 